\title{
Three-Dimensional Thermo Fluid Analysis of Large Scale Electric Motor
}

\author{
DEBASISH BISWAS, MASARU ISHIZUKA* and HIDEO IWASAKI \\ Mechanical Systems Laboratory, Research and Development Center, Toshiba Corporation, \\ 1, Komukai-Toshiba-Cho, Saiwai-Ku, Kawasaki, 210-8582, Japan
}

(Received 13 April 1999; In final form 21 May 1999)

\begin{abstract}
In the present work, the flow and temperature fields in large scale rotating electric motor are studied by solving the Navier-Stokes equations along with the temperature equation on the basis of finite difference method. All the equations are written in terms of relative velocity with respect to the rotating frame of reference. Generalized coordinate system is used so that sufficient grid resolution could be achieved in the body surface boundary layer region. Differential terms with respect to time are approximated by forward differences, diffusion terms are approximated by the implicit Euler form, convection terms in the Navier-Stokes equations are approximated by the third order upwind difference scheme. The results of calculation led to a good understanding of the flow behavior, namely, the rotating cavity flow in between the supporting bar of the motor, the flow stagnation and region of temperature rise due to flow stagnation, etc. Also the measured average temperature of the motor coil wall is predicted quite satisfactorily.
\end{abstract}

Keywords: Large scale electric motor, Rotor coil support, Heat flux, Computational fluid dynamics (CFD), Generalized coordinate system, Rotating cavity flow, Upwind difference scheme

\section{INTRODUCTION}

In recent days, the application of computational fluid dynamics (CFD) to the prediction of flow and thermal phenomena is getting more and more important. Over past twenty years steady progress has been made in the development of fluid flow and thermal behavior analyses for turbomachineries. The eventual goal of these analyses is a time accurate model of the three-dimensional flow and thermal phenomena.

In the open literature, there are a very large number of published papers regarding the analyses on turbomachinery flow problem which have been compared to experimental data reported by Weinberg et al. (1986), Chima (1987), Hah (1989) and Boyle (1991). These studies included twoand three-dimensional calculations using the

*Corresponding author. Tel.: 81-44-549-2331. Fax: 81-44-520-2057. E-mail: masaru.ishiduka@toshiba.co.jp. 
Navier-Stokes equations with some sorts of turbulence modeling. These analyses of flow could be used to study many of the fluid dynamic phenomena in turbomachinery. However, in order to use a computer code as an engineering tool to provide necessary information regarding the flow and thermal behavior it is essential to verify the prediction reliability of the code developed on the basis of recent improved numerical scheme and physics based modeling by comparing with experimental data of precious measurement accuracy.

Among existing numerical methods for solving incompressible Navier-Stokes equations, the finite difference method which uses the primitive variables, i.e., the velocities and the pressure as unknown variables prevails because of it's applicability to three-dimensional turbulent flows. For instance, the artificial compressibility method originally proposed by Chorin (1967) is quite well known for solving incompressible flows. Recently, this method is improved by introducing the efficient numerical techniques such as the linearization of Steger and Kutler (1977), diagonalization of Chang et al. (1988), etc. and also extended to the orthogonal and generalized curvilinear coordinate system reported by Hartwich and Hsu (1988) and Dick (1989). The other widely used method for solving unsteady incompressible Navier-Stokes equations is the well known MAC (Marker and Cell) method developed by Harlow and Welch (1965). In this method, a Poisson-type equation for pressure is derived on the basis of momentum equations and this Poisson equation for the pressure is employed in such a way that the continuity equation is satisfied at each time step. And the occurrence of spurious error (checkerboard oscillation of pressure) is suppressed by using a rectangular staggered grid topology. Recently, this method is extended to the orthogonal and generalized curvilinear coordinate grid topology.

In the thermal design of electric motor, it is very much essential to have a clear understanding of the complicated flow and thermal behavior in the electric motor. However, it is very difficult and expansive to gather information on flow and temperature field in the motor based on experiments. Therefore, in the present work, an attempt is made to develop a three-dimensional viscous flow solver for rotating flows to predict the flow and thermal behavior in a practical large scale electric motor with rotation. The coils of electric motor is typically cooled by air introduced from both ends. Because of the current flow through the coils, the coil surface get heated due to coil resistance. And the coil surface temperature need to be kept below certain critical temperature, since the insulation materials within the coil cannot withstand very high temperature. In order to keep the coil surface temperature below a critical level, the coil need to be cooled in an efficient manner. In the present case, the coils are cooled efficiently by air supplied from blower. The flow, in the present work is considered as incompressible, since the flow Mach number in the present case is quite less than 0.3 and MAC method is used to compute the flow and temperature field. Incompressible Navier-Stokes equations are solved directly without using any turbulence model. The validity of this numerical method is verified by the first author by comparing with experimental data on compressor blade cascade of precious measurement accuracy. In this flow problem of electric motor, the convection of heat from the heated coil surface to the fluid need to be considered. In this work, this problem is solved by coupling the Navier-Stokes equations with the thermal transport equation of fluid. As a boundary condition heat flux at the coil wall is specified to obtain the coil surface temperature. In order to take into account the rotational effect, the NavierStokes equations are written in terms of relative velocity with respect to the rotational frame of reference by including the angular momentum in the source term of the momentum equations. All the equations are written in the generalized coordinate system so that a sufficient number of grid points could be distributed in the body surface boundary layer region. All the equations are written in the form of finite difference equations. A numerical technique which suppresses non-linear instability for calculations of high-Reynolds number flows is 
used. In this technique, differential terms with respect to time are approximated by forward differences, diffusion terms are approximated by the implicit Euler form, convection terms in the Navier-Stokes equations are approximated by the explicit third order upwind difference scheme proposed by Kawamura and Takami (1986). Convective terms in the temperature equation are also approximated in the explicit third order upwind difference form and all other terms are approximated in the second order central difference form. The finite difference equations are solved by the point SOR (Successive Over Relaxation) method. The regular mesh system is employed to evaluate $P$, $u, v, w$ and $T$ at the mesh points. The present third order upwind scheme can also be used for quasiLES (Large Eddy Simulation) calculations in which the task of the subgrid-scale model to withdraw energy from the part of the spectrum was taken over by the dissipative numerical scheme. In fact, this effect can be achieved by numerical damping introduced by the third order upwind scheme. In the results and discussion section, computed flow and temperature field in the large scale electric motor is presented. The results led to a good understanding of the complicated flow behavior and the temperature distribution in the coil passage.

\section{MATHEMATICAL MODEL}

\subsection{Basic Three-Dimensional Equations}

The basic equations are the unsteady NavierStokes equations for three-dimensional incompressible rotational flow which in term of relative velocity with respect to the rotational reference frame can be written as follows:

Continuity equation

$$
\frac{\partial u_{i}}{\partial x_{i}}=0 \quad(I=1,2,3),
$$

Momentum equation

$$
\frac{\partial u_{i}}{\partial t}+u_{j} \frac{\partial u_{i}}{\partial x_{j}}=-\frac{1}{\rho} \frac{\partial P}{\partial x_{i}}+v \frac{\partial^{2} u_{i}}{\partial x_{j} \partial x_{j}}+H
$$

Temperature equation

$$
\frac{\partial T}{\partial t}+u_{j} \frac{\partial T}{\partial x_{j}}=k \frac{\partial^{2} T}{\partial x_{j} \partial x_{j}}+Q
$$

where in the case of $z$ as the axis of rotation, the source term $H$ in the momentum equation (2) can be written as follows:

$$
H=\varpi\left|\begin{array}{c}
x \varpi+2 v \\
y \varpi-2 u \\
0
\end{array}\right| .
$$

In Eqs. (1)-(3), $i$ and $j$ varies from 1 to 3 i.e. $x, y$ and $z$ directions, respectively. $\rho, u_{i}, P, v$ and $\omega$ are density, components of velocity, pressure, kinematic viscosity and angular velocity respectively. $Q$ in Eq. (3) represents the heat flux. These equations are transformed in the generalized curvilinear coordinates and are solved by the finite difference method following MAC (Marker and Cell) technique. The metric relations between $x_{\xi}, x_{\eta}, x_{\zeta}$ and $\xi_{x}, \eta_{x}, \zeta_{x}$, etc. in the transformation from cartesian coordinates $(x, y, z)$ to general curvilinear coordinates $(\xi, \eta, \zeta)$ can be found in the CFD book of Anderson et al. (1984) and therefore will not be repeated here.

\subsection{Derivation of Two-Dimensional Flow Equations}

In this section the derivation of two-dimensional incompressible viscous flow equations will be presented in detail. Extension to three-dimensional form is quite straight forward.

Following MAC method, the Poisson equation for pressure $P$ can be derived by taking the divergence of Eq. (2) as follows:

$$
\nabla^{2} P=-\operatorname{div}(v \cdot \nabla) v+R
$$

where $v$ represents velocity vector and $R$ is defined as

$$
R=-\frac{\partial D}{\partial t}+\frac{1}{R e} \nabla^{2} D, \quad D=\nabla \cdot v,
$$


where $R e$ in Eq. (6) is Reynolds number which comes from the non-dimensional form of Eq. (2). $R$ in Eq. (6) is identically zero on the basis of continuity equation, however, it is retained here as corrective term in order to prevent the accumulation of numerical errors. $R$ also helps stabilizing the computation and it goes near zero at convergence.

In the transformed coordinates, the Poisson equation for pressure and the two components of momentum equation can be written as follows:

$$
\begin{aligned}
\nabla^{2} P= & -\frac{1}{J^{2}}\left\{\left(y_{\eta} u_{\xi}-y_{\xi} u_{\eta}\right)^{2}\right. \\
& +2\left(x_{\xi} u_{\eta}-x_{\eta} u_{\xi}\right)\left(y_{\eta} v_{\xi}-y_{\xi} v_{\eta}\right) \\
& \left.+\left(x_{\xi} v_{\eta}-x_{\eta} v_{\xi}\right)^{2}\right\} \\
& +\frac{1}{(J \Delta t)}\left(y_{\eta} u_{\xi}-y_{\xi} u_{\eta}+x_{\xi} v_{\eta}-x_{\eta} v_{\xi}\right), \\
u_{t} & +\left(\frac{u y_{\eta}-v x_{\eta}}{J}\right) u_{\xi}+\left(\frac{v x_{\xi}-u y_{\xi}}{J}\right) u_{\eta} \\
& =-\frac{y_{\eta} p_{\xi}-y_{\xi} p_{\eta}}{J}+\frac{1}{R e} \nabla^{2} u, \\
v_{t} & +\left(\frac{u y_{\eta}-v x_{\eta}}{J}\right) v_{\xi}+\left(\frac{v x_{\xi}-u y_{\xi}}{J}\right) v_{\eta} \\
& =-\frac{x_{\xi} p_{\eta}-x_{\eta} p_{\xi}}{J}+\frac{1}{R e} \nabla^{2} v .
\end{aligned}
$$

In the two-dimensional case Jacobian of transformation $J$ is written as

$$
J=x_{\xi} y_{\eta}-x_{\eta} y_{\xi}
$$

Euler backward implicit scheme is used to integrate Eq. (2) (or (8) and (9)) as a remedy for numerical instability. According to this scheme, in the two-dimensional case the time integration is performed as follows:

$$
\frac{v^{n+1}-v^{n}}{\Delta t}+\left(v^{n} \cdot \nabla\right) v^{n+1}=-\nabla P+\frac{1}{R e} \nabla^{2} v^{n+1},
$$

where the second term of the left hand side is linearized for $v^{n+1}$ as follows:

$$
\left(v^{n+1} \cdot \nabla\right) v^{n+1}=\left(v^{n} \cdot \nabla\right) v^{n+1} .
$$

All spatial derivatives except non-linear term of Poisson equation and Navier-Stokes equations are approximated by central difference. The diffusion terms are approximated by the implicit Euler form, while the convective term in the momentum equations and temperature equation are approximated by the modified explicit third order upwind difference scheme originally proposed by Kawamura and Takami (1986), where, the third order error term is eliminated and the final form takes the form as follows:

$$
\begin{aligned}
& \left(f \frac{\partial u}{\partial \xi}\right)_{i j}=f_{i j}\left(\frac{-u_{i+2, j}+8\left(u_{i+1, j}-u_{i-1, j}\right)+u_{i-2, j}}{12 \Delta \xi}\right) \\
& +\alpha\left|f_{i j}\right| \frac{\left(u_{i+2, j}-4 u_{i+1, j}+6 u_{i j}-4 u_{i-1, j}+u_{i-2, j}\right)}{12 \Delta \xi}
\end{aligned}
$$

where $\alpha=3$.

The finite difference equations are obtained by discretizing Eqs. (7)-(9) on the basis of the above expression. In the present work they are solved by the point SOR (Successive Over Relaxation) method. Regular mesh system is employed to evaluate $P, u, v, w$ and $T$ at each individual grid points.

\subsection{Computation Flow Chart}

Calculation start with a specified initial velocity, pressure and temperature field at time $t=0$, the Poisson equation is solved first to get pressure $P$.

Then by substituting these pressure values into momentum equations, the velocity components at the next time step is computed by solving $v_{t}$ and the temperature is calculated next. The process is repeated until the required converged solution is obtained.

\section{COMPUTATION OUTLINE}

In this section information regarding the grid topology, convergence criteria, computation time 
and specification of boundary conditions, etc. will be discussed.

\subsection{Grid Topology and Convergence Criteria}

The coils of electric motor considered in the present study is cooled by air introduced from both ends. Although a six poles large practical electric motor is considered as the test case in the present study, in order to have some understanding of the configuration of electric motor, a schematic view of a typical four poles motor is presented in Fig. 1. It can be seen from this figure that this motor has four coils with supporting structure in between coils which rotate with respect to the flow axis. Because of the flow of current through the coil, the coil surface get heated. And the coil surface temperature need to be kept below certain critical temperature, since the insulation materials within the coil cannot withstand very high temperature. In order to keep the coil surface temperature below a critical level, the coil need to be cooled in an efficient manner. In the present case, the coils are cooled efficiently by air from blower which flows through the gap

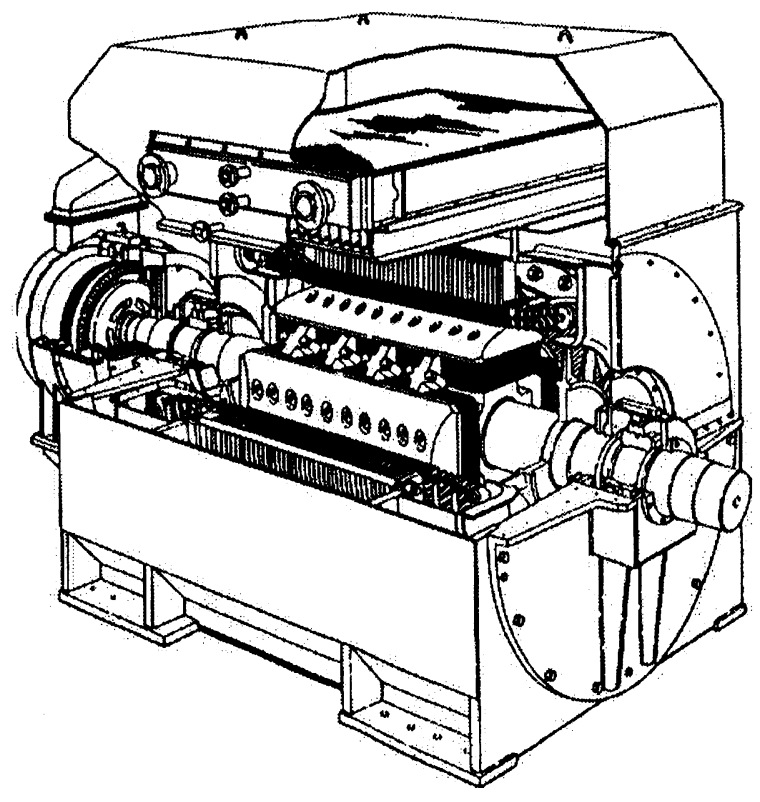

FIGURE 1 Schematic view of electric motor. between rotor coils from each end, meet near the mid of flow axis and then flows out through small holes of outer stator walls. This air is used for cooling the stator coil also. The computation model of the six poles large scale practical electric motor used in the present study is shown in Fig. 2. Since the flow is symmetric in the axial direction, only one half of the total axial length of the motor is considered as the computation domain. In order to take into account the effect of interaction between the stator and the rotor, computations are carried out using two zones. Rotor section is represented by the first zone and stator section is represented by the second zone. The interface boundary between these two zones is the junction between the stator inner boundary and rotor outer boundary. Since the coils in the motor are set in a periodic manner, and the six poles motor are six-fold symmetry, $60^{\circ}$ periodic sector model is used. Two rotor coils were separated by a gap of approximately, $20^{\circ}$ and the cooling holes for the passage of air from the rotor section through the stator coil were located with an axial gap of $40 \mathrm{~mm}$.

As it can be seen from Fig. 2, square shaped holes of length $10 \mathrm{~mm}$ and angular width of approximately, $2^{\circ}$ are used in the present study. In order to compute the flow in a complicated configuration like electric motor with rotation and strong threedimensional effect and the presence of blockage (coil support structure) in the coil flow path, computation grids are formed using a body fitted coordinate (BFC) grid generator. In the computation zone (one), $69 \times 151 \times 157$ grid points are used in $r, \theta$ and $z$ direction, respectively. In the computation zone (two) $16 \times 151 \times 157$ grid points are used. In Fig. 3 is presented a schematic illustration depicting the grid structure of the computation zone one. It can be observed here that fine grids are used in the coil surface region to properly simulate the boundary layer flow and thermal behavior. The non-dimensional distance $y+$ from the coil surface to the nearest grid points were of the order of 1 .

The convergence criteria for the Poisson equation of pressure was set as the residue $\leq 10^{-5}$ and that for $r, \theta$ and $z$ components of velocity and 


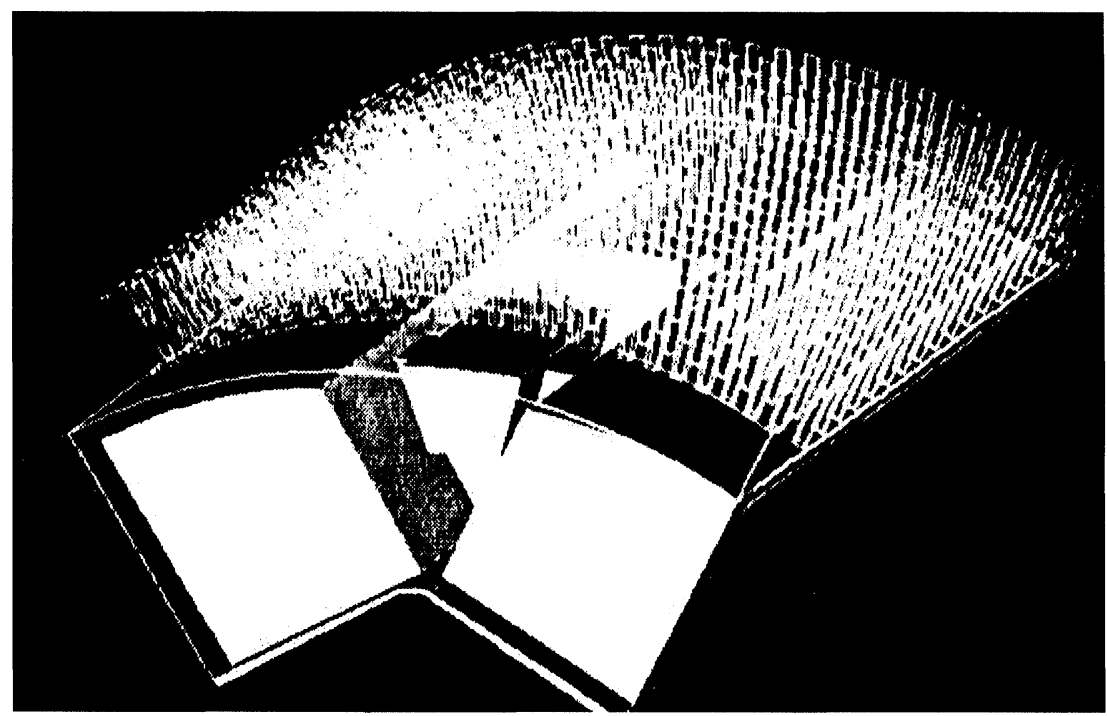

FIGURE 2 Computational model of electric motor

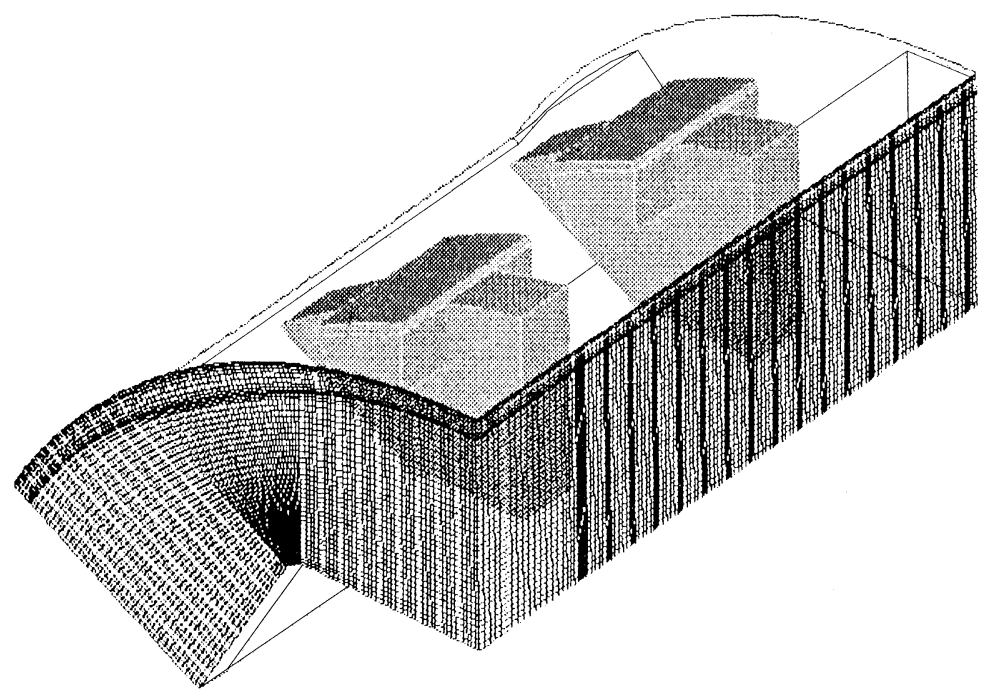

FIGURE 3 Computational grid of electric motor.

temperature were set as the residue $\leq 10^{-6}$. In order to meet the above mentioned convergence criteria, for Poisson equation of pressure less than 100 iterations were required, whereas for the rest of the parameters less than 20 iterations were required. Approximately 5000 time iterations were required to capture the flow and thermal phenomena. By monitoring pressure values at some important locations of the coil surface it is observed that the flow is regular oscillatory in nature. The oscillatory nature of the flow is presented in Fig. 11. Calculations were carried out using Cray T916 supercomputer with constant time step. The order of the non-dimensional time step was 0.02 . The present computation used about $100 \mathrm{Mw}$ memory and the overall computing costs in term of CPU time was approximately $10 \mathrm{~h}$ to capture the flow and thermal phenomena. 


\subsection{Boundary Conditions}

On the body surface velocity of fluid was set equal to zero, i.e.

$$
u=0 ; \quad v=0 \quad \text { and } \quad w=0 .
$$

It should be noted here that the zero velocity in case of rotor coils does not imply zero absolute velocity components, but rather zero relative velocity components. The pressure on the body surface was determined on the basis of Neuman type boundary condition i.e. the pressure gradient normal to the body surface was set equal to zero,

$$
\frac{\mathrm{d} p}{\mathrm{~d} n}=0 .
$$

Wall temperature was calculated on the basis of wall heat flux and the calculated fluid temperature. At the inlet of rotating coil, relative velocity and fluid temperature was specified and, at the symmetry boundary mirror image was considered to specify the boundary conditions.

\section{RESULTS AND DISCUSSIONS}

The flow Reynolds number for the present computation was about $2 \times 10^{5}$, which was defined in terms of inlet relative flow velocity and the radius of the rotor coil. The rotational speed of the coil was $150 \mathrm{rpm}$ and wall heat flux of the coil was $9950 \mathrm{~W} / \mathrm{m}^{2}$. The inlet air temperature was $303 \mathrm{~K}$.
In Fig. 4 are presented the computed vector diagram in the $z-\theta$ plane of rotor coil at three different radial locations. The flow direction in Fig. 4 is from right to left. In Fig. 4(a)-(c) are shown the velocity vector near the rotor hub, slightly away from the hub and near the edge of the supporting structures which are located in between the coils, respectively. It can be seen from Fig. 4 that there developed a re-circulation zone near the coil leading edge for all the three radial locations. Also, Fig. 4 indicate that there occurs a rotating cavity like flow near and in between the supporting structure in the flow passage. At the symmetry boundary, where the flow from both ends meet, there occurs a flow stagnation region. Near the edge of the supporting structure, the flow is divided by the presence of supporting structure and get accelerated at both of it's side. As a whole, Fig. 4 indicates that the presence of supporting structure in between the coils and the rotation made the flow highly unsteady, three-dimensional and complicated in nature.

In Fig. 5(a) and (b) are presented the velocity vector diagram in the $r-z$ plane, near the left and right coil, respectively. The flow direction is from right to left. It can be observed from this figure that near the hub, there occurs a rotating cavity like flow near and in between the coil supporting structures. Also, this figure indicates the flow direction at the rotor outer periphery i.e. at the interface between the rotor and the stator. The flow at the symmetry boundary is stagnated and is forced to flow toward the rotor outer periphery i.e. the small inlet hole of

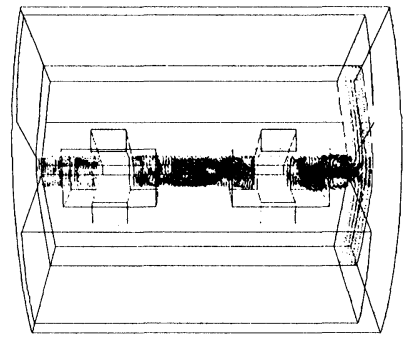

(a)

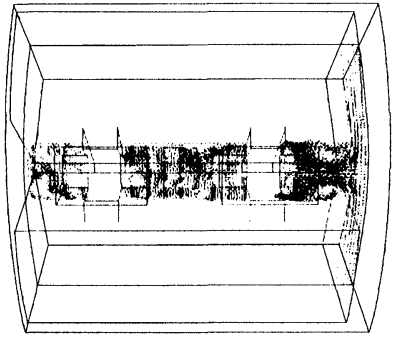

(b)

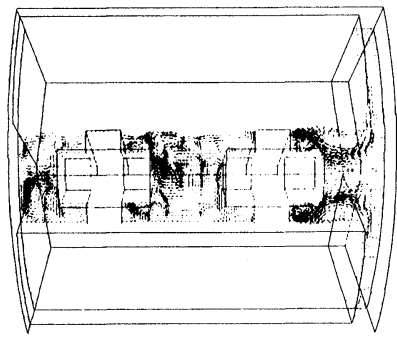

(c)

FIGURE 4 Computed velocity vector diagram in $z-\theta$ plane. 


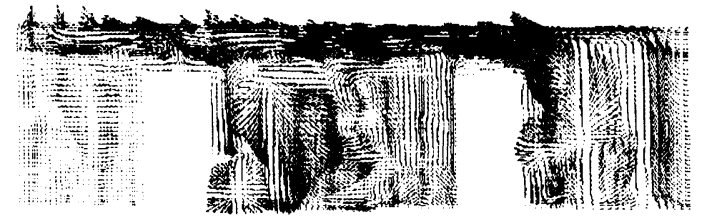

(a) left coil

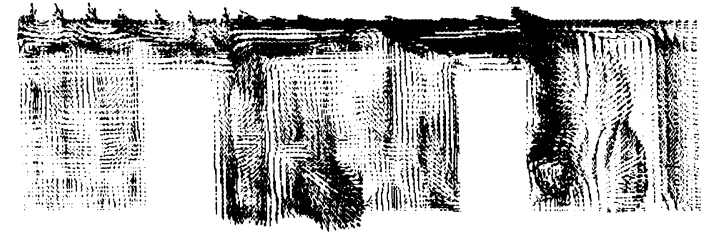

(b) right coil

FIGURE 5 Computed velocity vector diagram in $r-z$ plane.

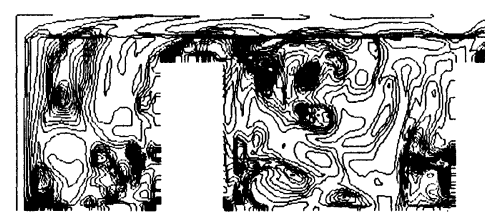

(a) left coil

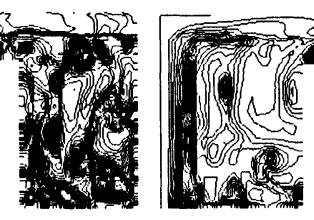
Mas

FIGURE 6 Computed temperature contour in $r-z$ plane.

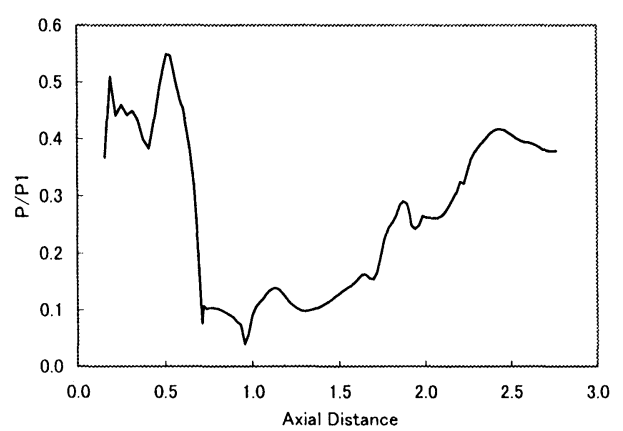

(a) left coil

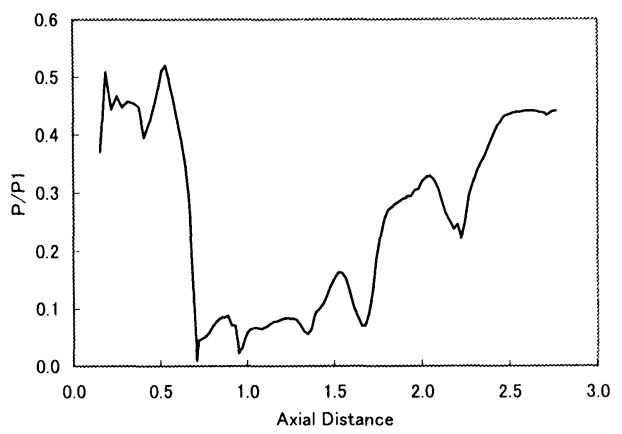

(b) right coil

FIGURE 7 Axial variation of surface pressure near the supporting structure edge.

stator. There exist two re-circulation zones, one near the hub and other near the rotor's outer periphery, in the region between the symmetry boundary and second supporting structure of the coil.

In Fig. 6(a) and (b) are presented the temperature contour in the $r-z$ plane of the left and right coil surface, respectively. It can be observed from this figure that the temperature near the coil leading edge increases due to the existence of flow recirculation zone, however, due to the effect of rotation, high temperature region near left coil leading edge get diffused as compared to the right coil. Also, the temperature distribution in the region between the coil supporting structure of left coil differs from that of right coil. In the right coil a distinct high temperature region exist, however, in the left coil due to rotational cavity like flow this high temperature zone get diffused and a small high temperature region exists near the hub region of downstream supporting structure. In the region between the symmetry boundary and the downstream supporting structure the temperature at both the coil surface is quite high due to flow stagnation in this region.

In Fig. 7(a) and (b) are presented the axial variation of left and right coil surface pressure for a 


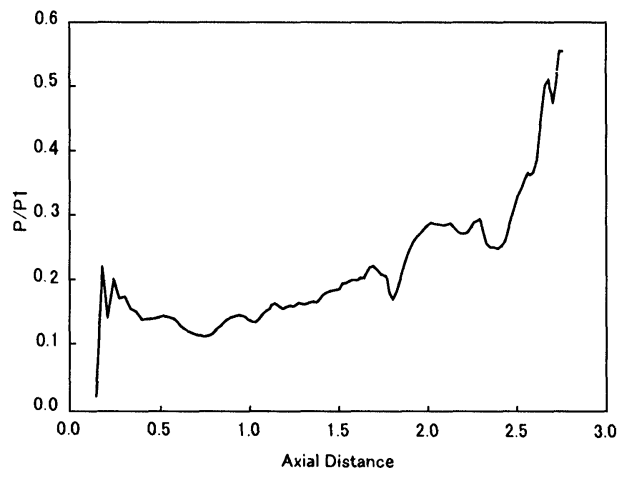

(a) left coil

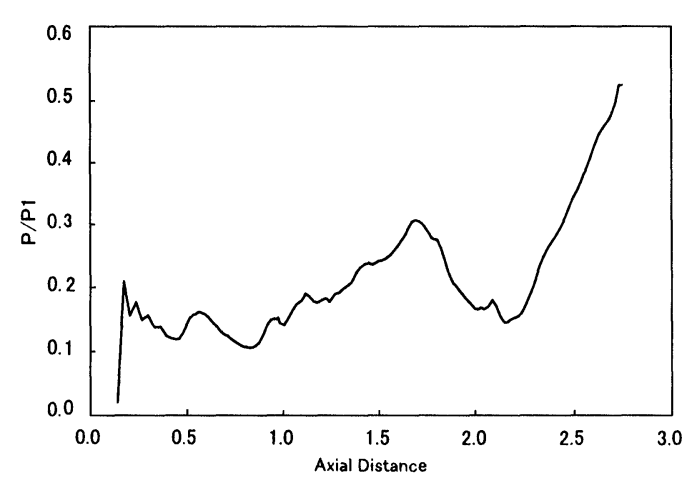

(b) right coil

FIGURE 8 Axial variation of coil top surface pressure.

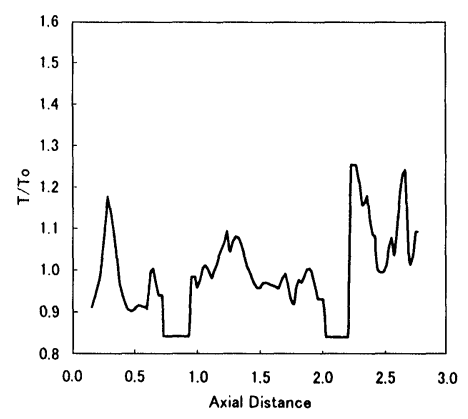

(a) near hub

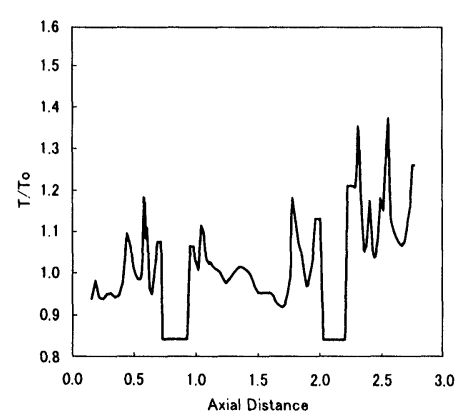

(b) in between hub and edge

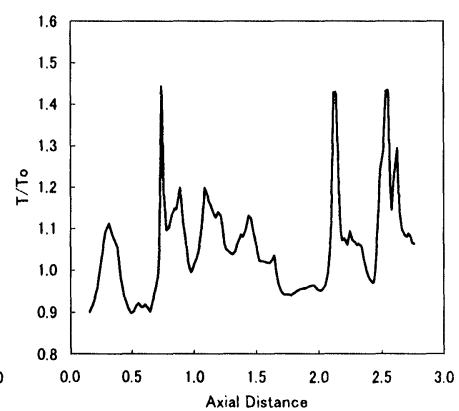

(c) near supporting structure edge

FIGURE 9 Axial variation of temperature on left coil.

radial location which corresponds to the edge of supporting structure, respectively. The surface pressure is non-dimensionalized with respect to the inlet pressure and axial distance is non-dimensionalized with respect to the rotor coil outer radius. It can be observed from this figure that at the leading edge of both the coils there occur an increase in pressure, followed by a decrease and again an increase due to the existence of flow re-circulation zone. Behind this re-circulation zone the pressure decreases abruptly due to flow acceleration and in the downstream region the pressure increases due to flow stagnation at the symmetry boundary. In the region covered by the non-dimensional axial distance of $0.7-1.7$ which corresponds to the region between two supporting structures, there occur some peak and trough in the pressure distribution curve of both the coils, which is thought to be due to the occurrence of cavity like rotating flow in this region. Also, in the case of right coil there occur a peak in the pressure distribution in this region due to rotational effect.

In Fig. 8(a) and (b) are presented the axial variation of pressure on the top surface of left and right coil, respectively. It can be observed from this figure that due to the presence of angular motion due to rotation, there occurs a peak in the pressure distribution in the right coil. Also, both Fig. 8(a) and (b) show an increase in pressure at the symmetry boundary due to flow stagnation.

In Figs. 9 and 10 are presented the axial variation of temperature for three different radial locations 


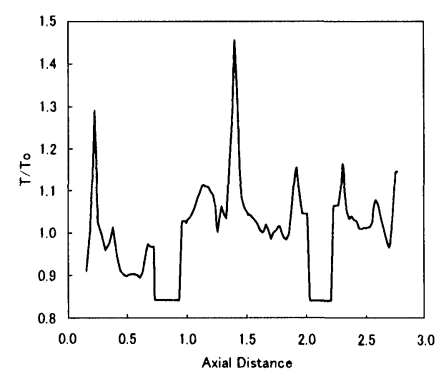

(a) near hub

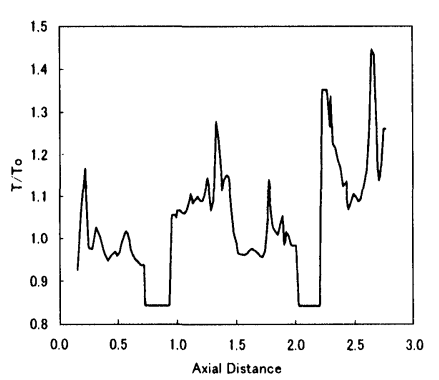

(b) in between hub and edge

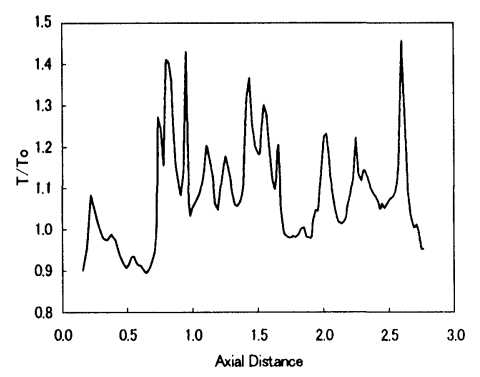

(c) near supporting structure edge

FIGURE 10 Axial variation of temperature on right coil.

(near the rotor hub, in between the hub and the supporting structure edge, and near the supporting structure edge) of left and right coil respectively. It can be observed from Fig. 10 that there occur a peak in the temperature distribution near the coil leading edge for all the radial location due to the existence of flow re-circulation zone. In the region between the supporting structure there occur successive peak and trough in the temperature distribution due to the complicated flow pattern i.e. the flow stagnation due to the presence of supporting structure, rotational cavity like flow and existence of flow re-circulation zone. In the case of temperature distribution in the left coil as shown in Fig. 8, the pattern is quite different from that observed in the case of right coil as shown in Fig. 9. In this case the peak in the temperature distribution near the coil leading edge due to the existence of re-circulation zone is quite distinct near the rotor hub and near the edge of supporting structure, however, in the region between the hub and the edge of supporting structure this peak is quite low and moved downstream due to angular motion. Also, the peak of the temperature distribution in the region between supporting structure in the case of left coil is lower than that in the case of right coil due to angular motion caused by rotation. Near the symmetry boundary the surface temperature of both the coils increases due to flow stagnation.

In Fig. 11 is presented the variation of pressure at the left and right coil side walls with time at a specific location. This location corresponds to an axial distance which is about $50 \%$ of the total length of computational domain (i.e. in between the supporting structure) and to mid-radial location (i.e. in between rotor hub and the rotor outer periphery). The time is non-dimensionalized with respect to reference velocity and length. It can be observed from this figure that there occur a phase lag in pressure fluctuation with time between the left and right coils and with the increase in time the peak and trough of pressure fluctuation at the left and the right coils is reversed i.e. the peak of pressure fluctuation at the left coil corresponds to the trough at the right coil due to the effect of rotation.

In Fig. 12 is presented the variation of pressure at the left and right coil top surface with time at the location which corresponds to about $50 \%$ axial distance of computational domain. Here also it can be observed that there occur a phase lag in pressure fluctuation with time between the left and right wall and with the increase in time the peak and trough of pressure fluctuation at the left and the right wall is reversed i.e. the peak of pressure fluctuation at the left wall corresponds to the trough at the right wall due to effect of rotation. Finally the computed and the measured average wall temperature of the coil is compared. The coil temperature was measured on the basis of electric resistance technique. In this method the average coil surface temperature was obtained by measuring the electric resistance in the coil. The calculated average temperature of coil surface was $325.8 \mathrm{~K}$ which is very satisfactory comparing the measured average surface temperature of $322.1 \mathrm{~K}$ in the practical coil. 


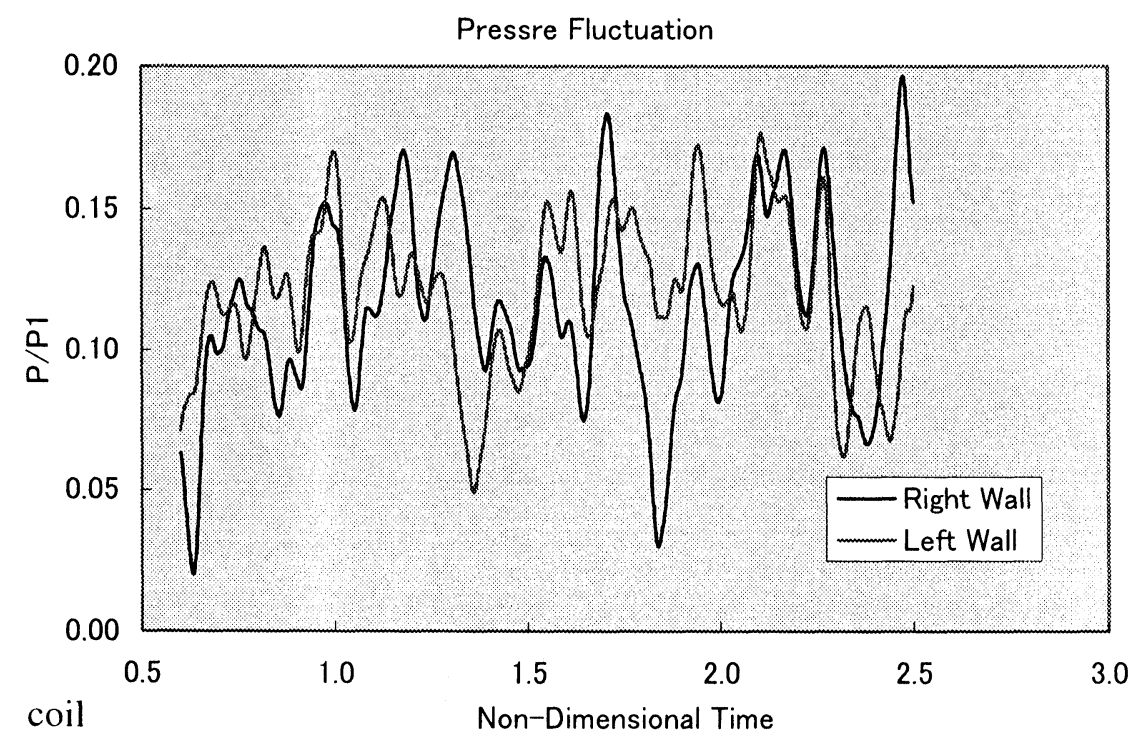

FIGURE 11 Coil side wall surface pressure fluctuation.

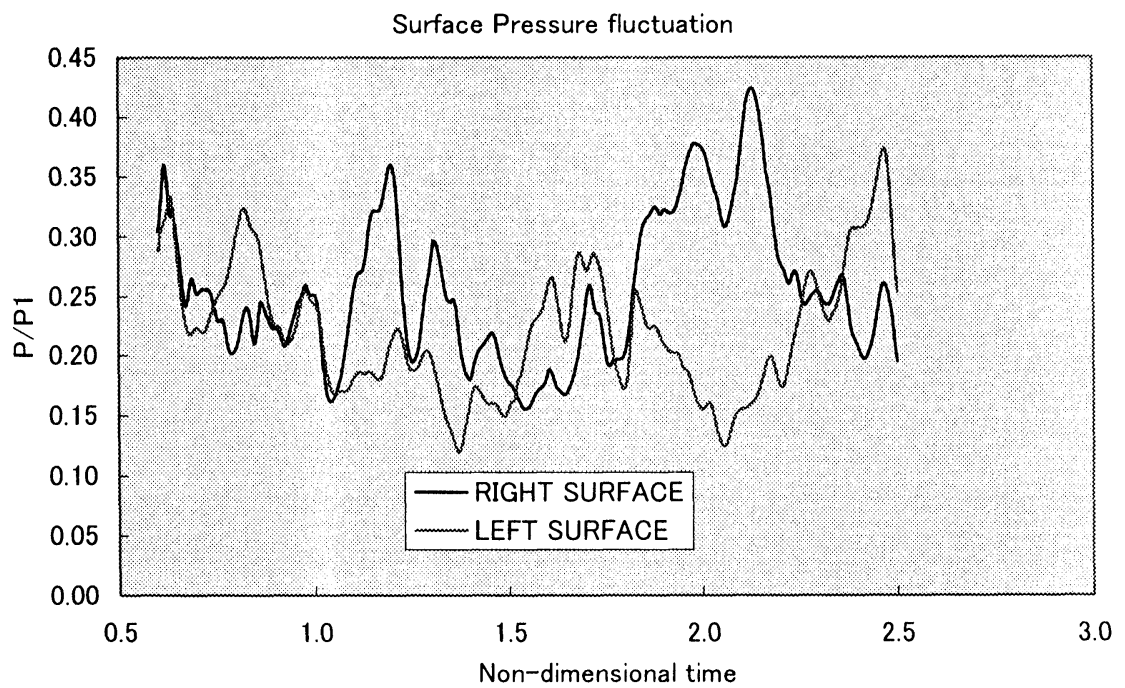

FIGURE 12 Coil top surface pressure fluctuation.

\section{SUMMARY}

In the present work, the flow and temperature fields in practical large scale rotating electric motor are studied by solving the Navier-Stokes equations along with the temperature equation on the basis of finite difference method without using any turbulence model.
The present scheme used in the computation led to a stable calculation even for such high-Reynolds number, complicated flow nature caused by the presence of obstacles and the rotational effect.

The results of calculation led to a good understanding of the flow and temperature field in the electric motor, namely, the existence of flow recirculation zone near the coil leading edge, rotating 
cavity like flow in between the supporting structure of the motor, the flow stagnation near the symmetry region and the region of coil surface temperature rise due to such complicated flow behavior.

The coil surface pressure fluctuation with time led to the understanding that there exists some phase lag in pressure fluctuation with time between the left and right wall and with the increase in time the peak and trough of pressure fluctuation at the left and the right wall is reversed i.e. the peak of pressure fluctuation at the left wall corresponds to the trough at the right wall due to effect of rotation.

It is observed that the present computation method could predict the average temperature of the coil surface quite satisfactorily. As a whole, the results of present computation could provide a useful information regarding the complicated flow behavior in the large scale electric motor to the designers.

\section{References}

Anderson, D.A. et al. (1984) Computational Fluid Mechanics and Heat Transfer, Hemisphere Publishing Corporation.

Boyle, R.J. (1991) Navier-Stokes analysis of turbine blade heat transfer, ASME Journal of Turbomachinery, 113.
Chang, J.L.C., Kwak, D. et al. (1988) Numerical simulation methods of incompressible flows and an application to the space shuttle main engine, International Journal of Numerical Mathematical Fluids, 8.

Chima, R.V. (1987) Explicit multigrid algorithm for quasithree-dimensional viscous flows in turbomachinery, Journal of Propulsion and Power, 3(5).

Chorin, A.J. (1967) A numerical method for solving incompressible viscous flow problems, Journal of Computation Physics, 2.

Dick, E.A. (1989) Multigrid method for steady incompressible Navier-Stokes equations based on partial flux splitting, International Journal of Mathematical Fluids, 9.

Dring, R.P. et al. (1993) A three-dimensional Navier-Stokes calculation applied to an axial compressor rotor and stator, ASME Paper No. 93-GT-113.

Hah, C. (1989) Numerical study of three-dimensional flow and heat transfer near the endwall of a turbine blade row, AIAA Paper No. 89-1689.

Harlow, F.H. and Welch, J.E. (1965) Numerical calculation of time dependent viscous incompressible flow of fluid with free surface, Physics Fluids, 8.

Hartwich, P.M. and Hsu, C.H. (1988) High resolution upwind schemes for the three-dimensional incompressible NavierStokes equations, AIAA Journal, 26.

Kawamura, T. and Takami, H. (1986) Computation of high Reynolds number flow around a circular cylinder with surface roughness, Fluid Dynamic Research, 1.

Steger, J.L. and Kutler, P. (1977) Implicit finite difference procedures for the computation of vortex wakes. AIAA Journal, 15.

Weinberg, B.C. et al. (1986) Calculation of two- and threedimensional transonic cascade flow fields using the NavierStokes equations, ASME Journal of Engineering for Gas Turbines and Power, 108 


\section{ait \\ ENERGY MATERIALS}

M A N E Y publishing

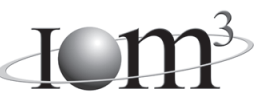

\section{Materials Science \& Engineering for Energy Systems}

Maney Publishing on behalf of the Institute of Materials, Minerals and Mining

The Institute of Materials, Minerals \& Mining

Economic and environmental factors are creating ever greater pressures for the efficient generation, transmission and use of energy. Materials developments are crucial to progress in all these areas: to innovation in design; to extending lifetime and maintenance intervals; and to successful operation in more demanding environments. Drawing together the broad community with interests in these areas, Energy Materials addresses materials needs in future energy generation, transmission, utilisation, conservation and storage. The journal covers thermal generation and gas turbines; renewable power (wind, wave, tidal, hydro, solar and geothermal); fuel cells (low and high temperature); materials issues relevant to biomass and biotechnology; nuclear power generation (fission and fusion); hydrogen generation and storage in the context of the 'hydrogen economy'; and the transmission and storage of the energy produced.

As well as publishing high-quality peer-reviewed research, Energy Materials promotes discussion of issues common to all sectors, through commissioned reviews and commentaries. The journal includes coverage of energy economics and policy, and broader social issues, since the political and legislative context influence research and investment decisions.

\section{CALL FOR PAPERS}

Contributions to the journal should be submitted online at http://ema.edmgr.com

To view the Notes for Contributors please visit: www.maney.co.uk/journals/notes/ema

Upon publication in 2006, this journal will be available via the Ingenta Connect journals service. To view free sample content online visit: www.ingentaconnect.com/content/maney

For further information please contact:

Maney Publishing UK

Tel: +44 (0)113 2497481 Fax: +44 (0)1132486983 Email: subscriptions@maney.co.uk

or

Maney Publishing North America

Tel (toll free): 8662975154 Fax: 6173546875 Email: maney@maneyusa.com

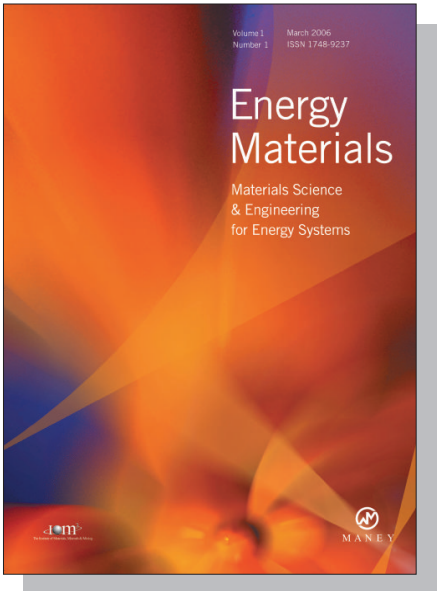

EDITORS

Dr Fujio Abe

NIMS, Japan

Dr John Hald, IPL-MPT, Technical University of Denmark, Denmark

Dr R Viswanathan, EPRI, USA

\section{SUBSCRIPTION INFORMATION}

Volume 1 (2006), 4 issues per year

Print ISSN: 1748-9237 Online ISSN: 1748-9245

Individual rate: $£ 76.00 / U S \$ 141.00$

Institutional rate: $£ 235.00 /$ US $\$ 435.00$

Online-only institutional rate: $£ 199.00 / U S \$ 367.00$

For special $\mathrm{IOM}^{3}$ member rates please email

subscriptions@maney.co.uk

\section{For further information or to subscribe online please visit www.maney.co.uk}



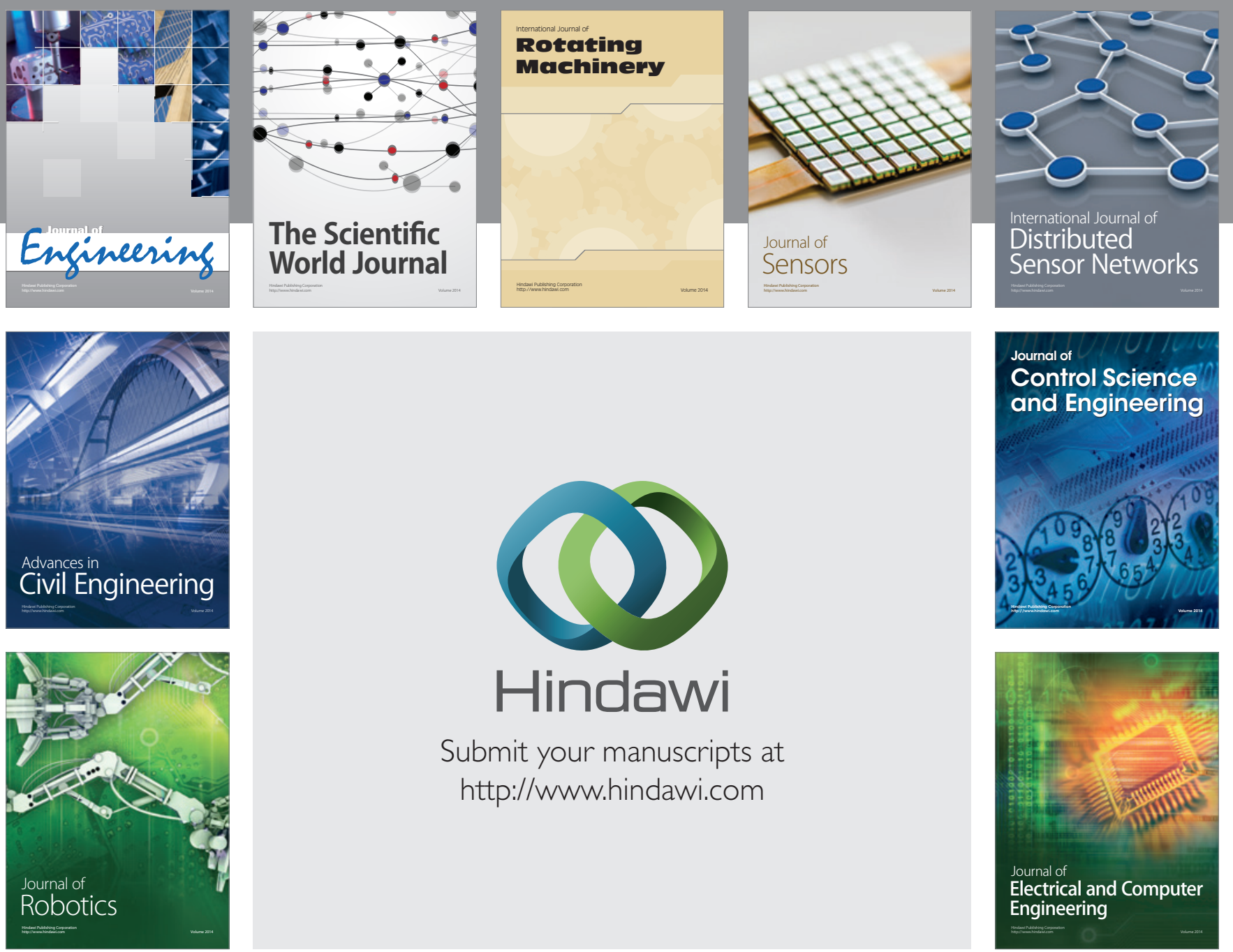

Submit your manuscripts at

http://www.hindawi.com
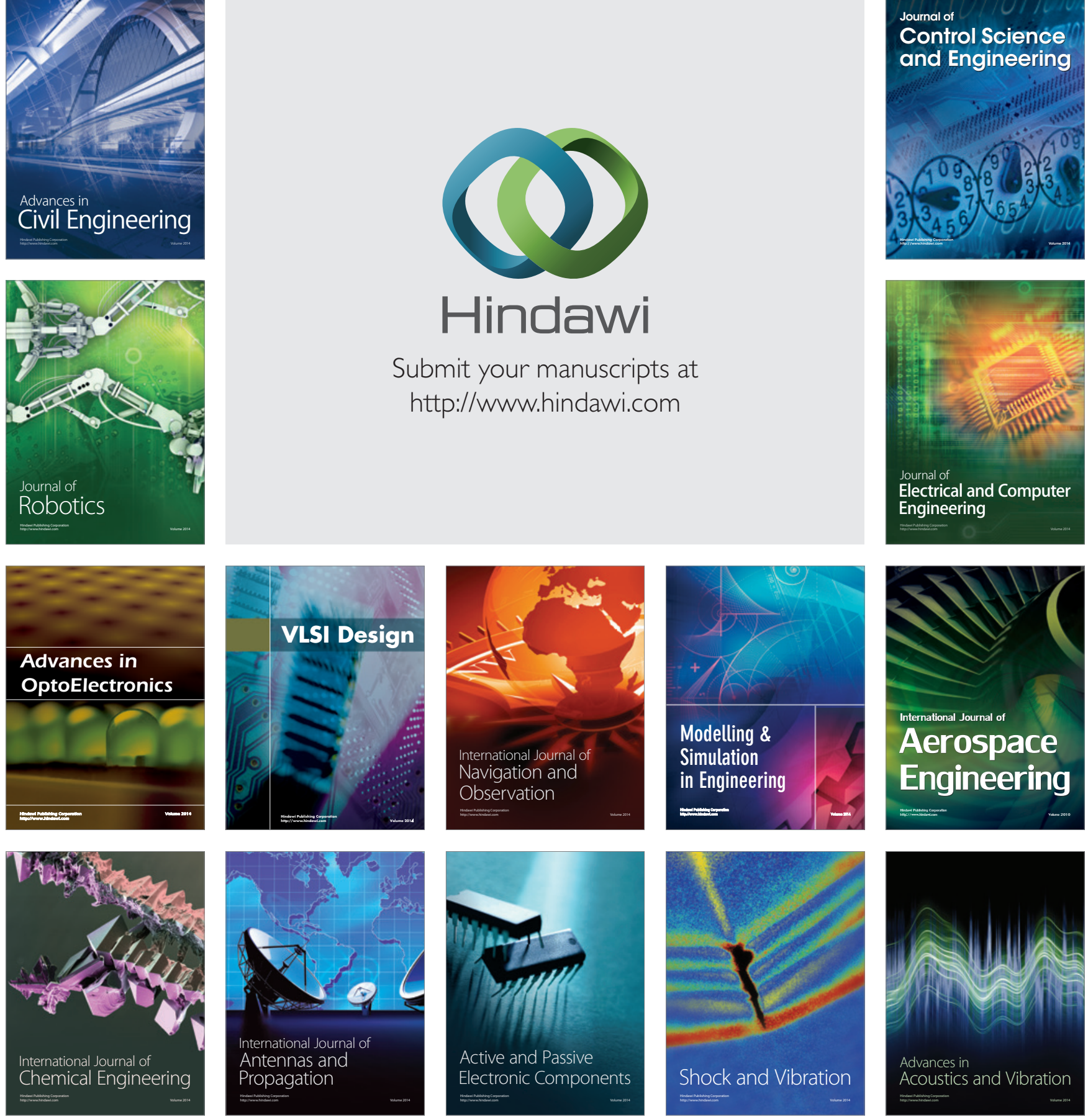